\title{
Low level laser therapy (Photobiomodulation therapy) for breast cancer-related lymphedema: a systematic review
}

G. David Baxter ${ }^{1 *}$, Lizhou Liu', Simone Petrich², Angela Spontelli Gisselman', Cathy Chapple, Juanita J. Anders ${ }^{3}$ and Steve Tumilty ${ }^{1}$

\begin{abstract}
Background: Breast cancer related lymphedema (BCRL) is a prevalent complication secondary to cancer treatments which significantly impacts the physical and psychological health of breast cancer survivors. Previous research shows increasing use of low level laser therapy (LLLT), now commonly referred to as photobiomodulation (PBM) therapy, for BCRL. This systematic review evaluated the effectiveness of LLLT (PBM) in the management of BCRL.

Methods: Clinical trials were searched in PubMed, AMED, Web of Science, and China National Knowledge Infrastructure up to November 2016. Two reviewers independently assessed the methodological quality and adequacy of LLLT (PBM) in these clinical trials. Primary outcome measures were limb circumference/volume, and secondary outcomes included pain intensity and range of motion. Because data were clinically heterogeneous, best evidence synthesis was performed.

Results: Eleven clinical trials were identified, of which seven randomized controlled trials (RCTs) were chosen for analysis. Overall, the methodological quality of included RCTs was high, whereas the reporting of treatment parameters was poor. Results indicated that there is strong evidence (three high quality trials) showing LLLT (PBM) was more effective than sham treatment for limb circumference/volume reduction at a short-term follow-up. There is moderate evidence (one high quality trial) indicating that LLLT (PBM) was more effective than sham laser for short-term pain relief, and limited evidence (one low quality trial) that LLLT (PBM) was more effective than no treatment for decreasing limb swelling at short-term follow-up.

Conclusions: Based upon the current systematic review, LLLT (PBM) may be considered an effective treatment approach for women with BCRL. Due to the limited numbers of published trials available, there is a clear need for well-designed high-quality trials in this area. The optimal treatment parameters for clinical application have yet to be elucidated.
\end{abstract}

Keywords: Low level laser therapy, Photobiomodulation, Breast cancer related lymphedema, Systematic review

\footnotetext{
* Correspondence: david.baxter@otago.ac.nz

${ }^{1}$ Centre for Health, Activity and Rehabilitation Research, School of

Physiotherapy, University of Otago, Dunedin, New Zealand

Full list of author information is available at the end of the article
} 


\section{Background}

With improvements in early detection, diagnosis, and treatment of breast cancer, as well as an increase in breast cancer incidence, the number of breast cancer survivors is growing [1]. It is estimated that nearly $82 \%$ of women survive at least 10 years after diagnosis in developed countries (e.g. Europe, United States, and Japan) [1]. In New Zealand, the 10-year survival rate is estimated to be $92 \%$ with regular mammogram detection [2].

While this is encouraging, a considerable number of breast cancer survivors suffer from secondary lymphedema due to cancer related treatments (surgery and/or radiation therapy). Despite efforts to reduce lymphedema rates with new surgical techniques like the sentinel node biopsy technique replacing the axillary dissection as a standard for clinically node negative patients, breast cancer related lymphedema (BCRL) remains a relevant concern. A recent systematic review estimated that more than one out of five women who survive breast cancer are affected by BCRL [3]. This is in concordance with New Zealand specific data; it was estimated that the incidence of BCRL in New Zealand is $23.3 \%$ [4]. BCRL has a significant impact on breast cancer survivors, including declined physical function and increased disability, which negatively affects quality of life [5-8]. While the mainstay of BCRL management approaches include compression garments, manual lymphatic drainage, and remedial exercises $[5,9,10]$, these interventions are usually time-consuming and poorly adherent (or unacceptable). There is a clear need for interventions to target the symptoms of BCRL and improve the wellbeing of these survivors.

Over the past two decades, low level laser therapy (LLLT) or photobiomodulation (PBM) has been promoted and researched for the management of BCRL. LLLT (PBM) is a non-invasive form of phototherapy that utilizes wavelengths of light between 650 and $1000 \mathrm{~nm}$ to deliver low irradiance and doses to the target tissue. It has been used to reduce inflammation, promote lymph vessel regeneration, improve lymphatic motility, and prevent tissue fibrosis [11-14]. It has been reported to be a safe technique [15]. Figure 1 illustrates an example of this technology.

Information on the basic mechanisms of LLLT (PBM) and a range of cellular effects have been demonstrated using a variety of cell types (fibroblasts; lymphocytes; osteoblasts; stem cells; smooth muscle cells) and in vitro [16-24]. These effects are the result of primary reactions involving absorption of specific wavelengths of light by components of the mitochondrial respiratory chain such as cytochromes, cytochrome oxidase, and flavin dehydrogenases; these result in changes in reduction-oxidation reaction (REDOX) status of cytoplasm and mitochondria, which in turn leads to increased levels of adenosine triphosphate (ATP) [25].

These primary reactions stimulate a cascade of secondary reactions at cellular level involving intracellular signalling and leading to stimulation of cytokine reactions, and nitric oxide (NO) production [17, 26]; release of growth factors [27-29]; up-regulation of ATP $[30,31]$, and increased metabolism, changes in REDOX signalling, increased reactive oxygen species (ROS) and therefore cell proliferation [30-32].In addition, stimulation of lymphatic vessels [33], and on lymphocytes [34] have been reported, as well as increases in local fluid circulation [35].

Previous literature reviews indicated promising effects of LLLT (PBM) for women with BCRL [15, 36, 37]. However, results were not robust due to a lack of formal synthesis methodology $[15,36]$, and the single metaanalysis did not perform subgroup analysis [37]. In order to address these issues, we aimed to conduct an updated systematic review of all available evidence from published clinical trials, including evidence from Chinese trials (with help of a Chinese collaborator), on the effectiveness of LLLT (PBM) for adult women with BCRL. Additionally, an assessment of treatment adequacy was carried out to examine the accuracy and clinical appropriateness of the treatment regimen of LLLT (PBM) in this area.
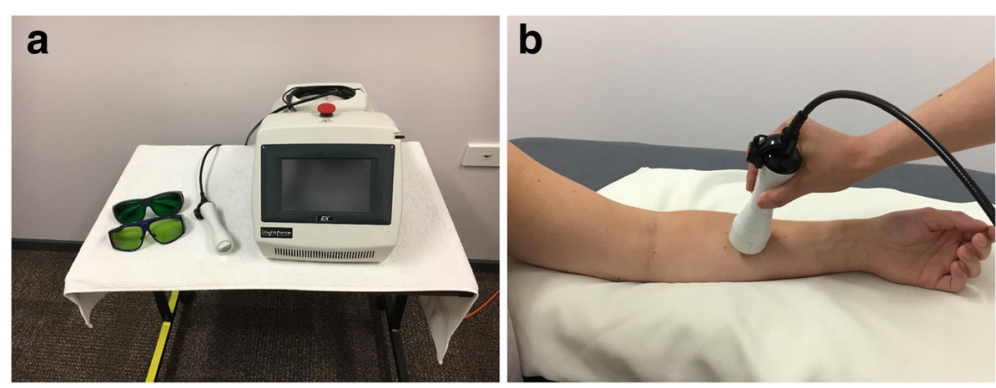

Fig. 1 Examples of the technique of LLLT (PBM). a A device of LLLT (PBM). b Applying the LLLT (PBM) treatment head over a forearm region. Abbreviations: LLLT, low level laser therapy; PBM, photobiomodulation 


\section{Methods}

\section{Protocol and registration}

The review protocol was not registered.

\section{Search strategy}

A comprehensive computer-aided literature search was undertaken in three English databases (PubMed, AMED, and Web of Science) and a Chinese database (CNKI) that includes grey literature (e.g. theses, conference proceedings), from their inception until November 2016. Search terms used were (cold laser OR laser OR laser light OR low-energy laser OR low-intensity laser OR low-level laser OR laser therapy OR photobiomodulation) AND (lymphedema OR lymphoedema OR swelling OR edema OR oedema) AND (breast cancer) with slight modifications for individual searches in each database (see Additional file 1 for search strategy). Additional articles were sought by manual screening of reference lists of all retrieved papers. Professionals working in the field were contacted to identify potential articles. Publication status was not restricted. No language restrictions were applied, provided there was an abstract available in English, as translation services were available.

\section{Inclusion criteria}

Studies were considered eligible for inclusion if they satisfied the following criteria:

(1) study design: clinical trials (e.g. randomized controlled trials (RCTs) and observational studies);

(2)population: adult women who were diagnosed with BCRL;

(3)intervention: LLLT/PBM therapy;

(4) control (if applicable): there was no restriction regarding the control group, including no treatment or waiting list, sham therapy, and conventional therapy as any active treatment other than LLLT (PBM);

(5) outcomes: clinically related outcome variables such as limb circumference/volume, pain intensity, range of motion, tissue resistance, tissue fluid, and subjective symptom.

\section{Exclusion criteria}

Studies that included patients with primary lymphedema or lymphedema secondary to pathologic entities other than breast cancer related treatment were excluded. Reviews, guidelines, surveys, commentaries, editorials, and letters were excluded.

\section{Study selection}

Two independent reviewers searched for potential articles by initially scanning the titles and abstracts to determine eligibility. Full papers were then reviewed for final inclusion. Differences between the reviewers were settled by discussion, and a third reviewer was consulted if differences persisted. Reviewers were not blinded to authors, institutions, publication journals, or study results.

\section{Data extraction}

Data were extracted independently by the two reviewers using two standardized spreadsheets (one for RCTs and one for observational studies) designed to record author(s) and year of publication, study population, intervention, control comparison (if applicable), cointervention, outcome measures, measurement timepoints, conclusions and funding sources. Consensus was reached by discussion. Authors of original studies were contacted if further information was needed.

\section{Assessment of methodological quality}

Methodological quality of included studies with RCT design was independently assessed by two reviewers using the physiotherapy evidence databases (PEDro) scale [38]. There was no blinding of study identification in this process. Before the assessment started, each item in the scale was intensively discussed to achieve consistency in the following procedure. Agreement level between the two reviewers was measured by the kappa statistic (kappa index less than 0.4 indicated poor agreement, 0.4 to 0.75 fair agreement, and over 0.75 excellent agreement) [39]. Again, consensus was reached through discussion. If a disagreement persisted, an independent decision was obtained from another collaborator. Since there are no accepted cutoff scores for the PEDro scale, a study was considered as high quality if the total score was 5 or higher $[15,36,40]$. The classification of quality was used to grade the strength of the evidence in data synthesis.

\section{Results synthesis}

Primary analysis was based solely on the results from RCTs. The control groups, outcome measures, and the time points of follow-ups, were grouped according to the following criteria as a priori:

(1)control comparisons: sham therapy which was physiologically inert; no treatment or waiting list; conventional therapy including compression bandages or garment, pneumatic compression pump, manual lymphatic drainage, complex decongestive therapy, and limb exercise;

(2)outcome measures: primary outcome: limb circumference/volume; secondary outcome: pain intensity and range of motion;

(3) time points: at discharge: immediately after end of all treatment sessions; short-term follow-up: $<6$ months after treatment; long-term follow-up: $\geq$ 6 months after treatment [41]. 
Outcomes of subgroup comparisons were summarized and evaluated. Meta-analysis was not performed due to the clinical heterogeneity and a limited number of included studies. Best evidence synthesis was conducted to generate final conclusions, taking into account the methodological quality, results of original studies, and numbers of RCTs that reported consistent findings (principal summary measures as effectiveness or non-effectiveness) [42]:

(1)Strong-consistent findings (more than $75 \%$ of RCTs report the same findings) among multiple high quality RCTs;

(2) Moderate-consistent findings among multiple low quality RCTs and/or one high quality RCT;

(3) Limited-one low quality RCT;

(4)Conflicting-inconsistent findings among multiple RCTs;

(5)No evidence from trials-no RCTs.

\section{Assessment of treatment adequacy}

LLLT (PBM) dosage parameters (e.g. wavelength, output power, power density (irradiance), energy density, and treatment area) of included studies (RCTs and observational studies) were used to judge the adequacy of treatment. Those parameters were compared to the World Association for Laser Therapy (WALT) guideline (https://waltza.co.za/documentation-links/recommendations/) [43]. Two reviewers who had extensive experience with research on laser applications independently assessed the adequacy and clinical appropriateness of the treatment dose, and resolved disagreement by discussion.

\section{Results}

\section{Study selection}

In total, 88 studies were identified through electronic and hand searches. After excluding duplicates and those which did not meet the inclusion criteria, 11 studies were finally included (see Additional file 2 for excluded articles). An observational trial conducted by Piller and Thelander was regarded as two studies in this review due to different follow-ups (preliminary results (1995) [44] and main findings (1998) [45]). LLLT (PBM) treatment adequacy was assessed by these eleven studies. Data on the seven RCTs of the 11 studies were included in the effectiveness analysis; the remaining four studies were excluded as observational studies (Fig. 2).

\section{Study characteristics}

Tables 1 and 2 present the main characteristics of the included RCTs and observational studies, respectively. All studies were published in English, and reported beneficial effects from LLLT (PBM). In the seven included RCTs, sample size ranged from 11 to 53 . All trials measured limb circumference/volume, three (42.9\%) measured pain intensity and range of motion. LLLT (PBM) was compared to sham laser therapy [46-48], conventional treatments including manual lymphatic drainage [49], pneumatic compression therapy [50] and

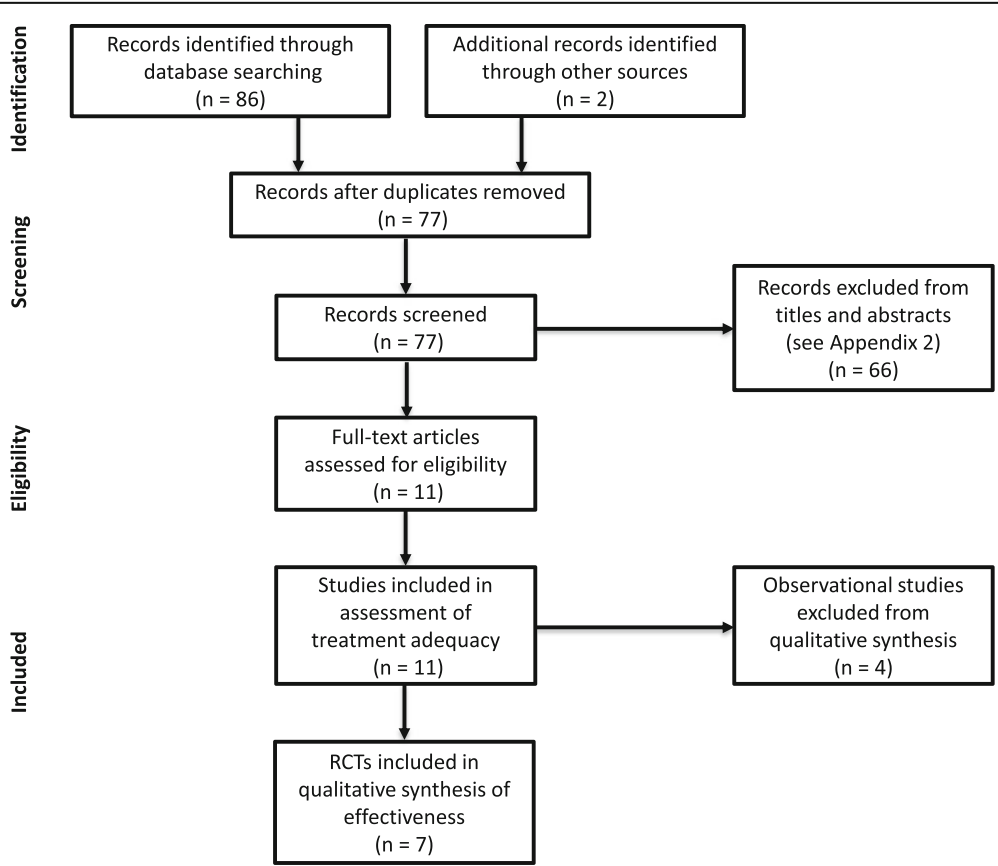

Fig. 2 Flow diagram of literature search 


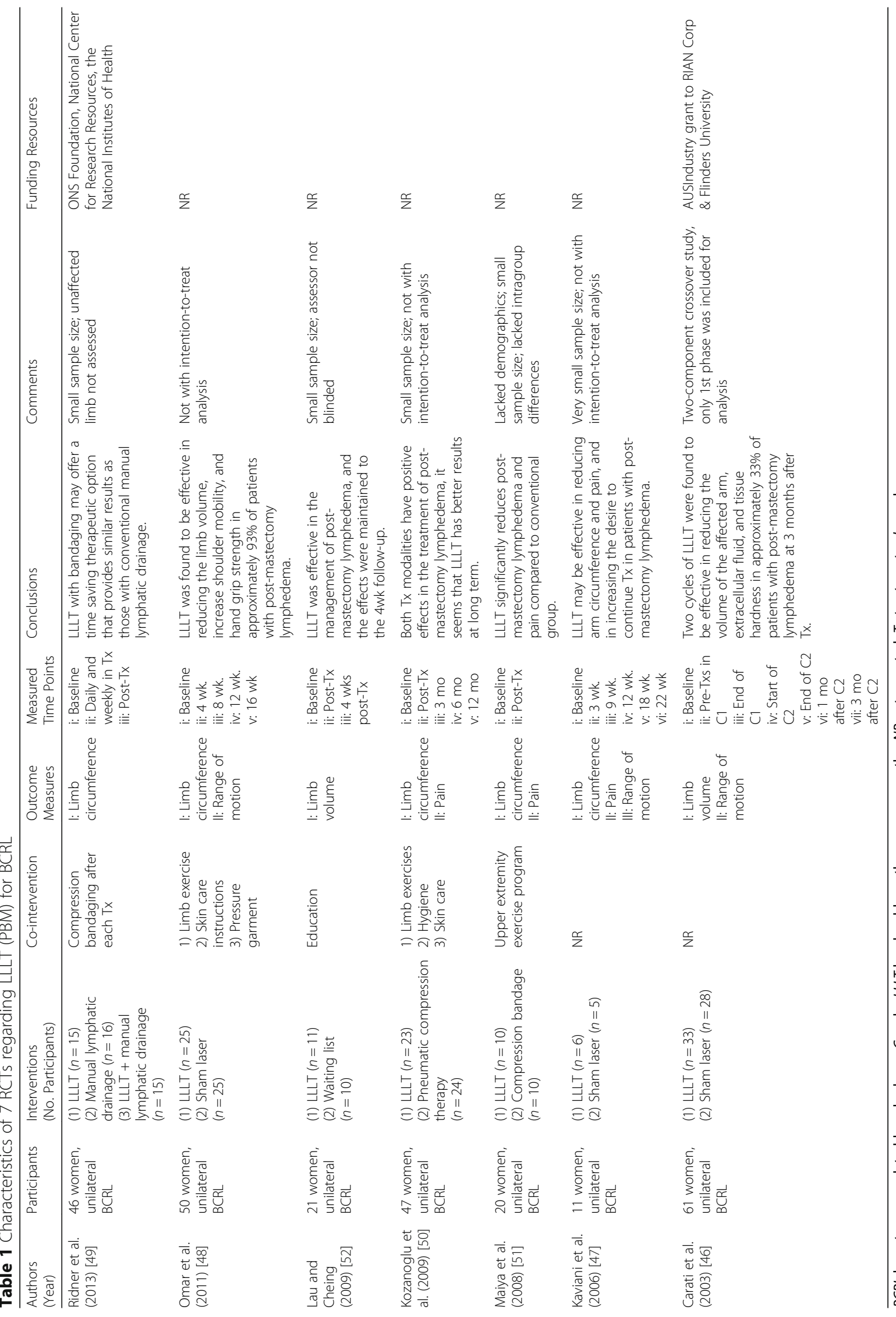




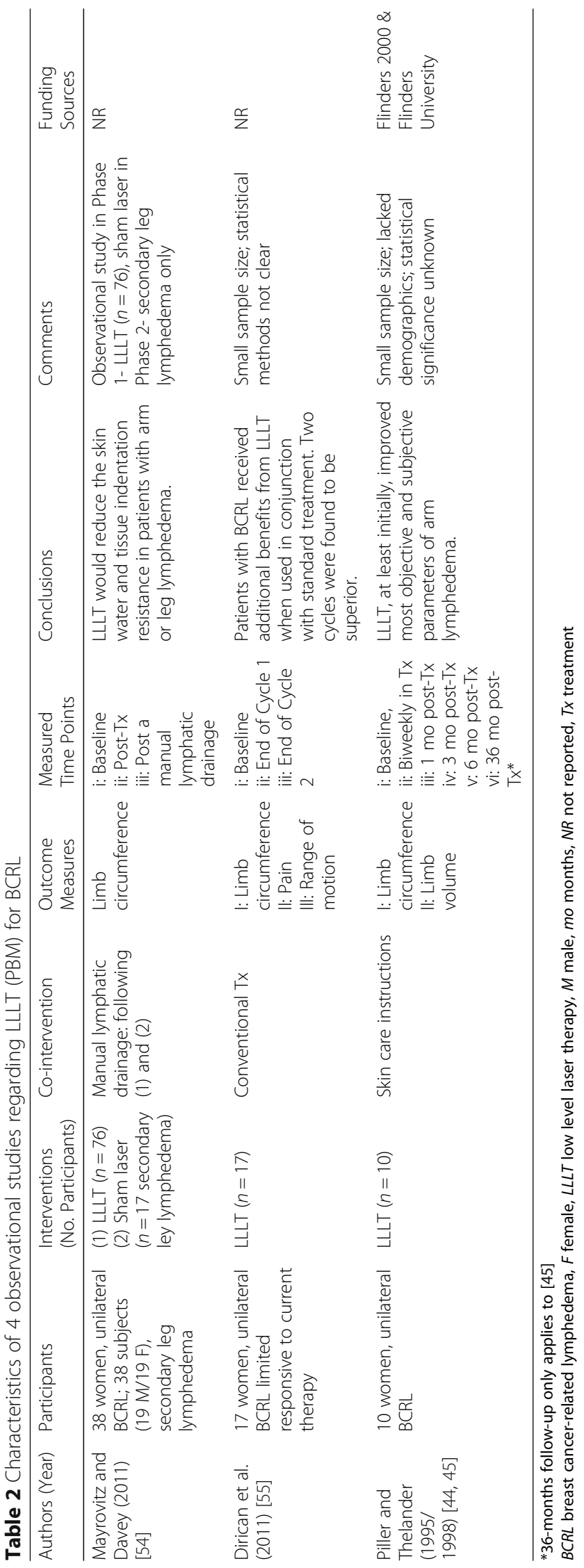


compression bandage [51], and a waiting list control [52]. Follow-up lengths varied widely amongst the RCTs. Two trials ended immediately after the treatment regimen [49, 51], two trials followed patients for 1 month $[48,52]$, and another two trial assessed patient outcomes up to 2 [47] and 3 months [46], respectively. One RCT extended assessment time to 12 months [50], which was considered as long-term follow-up.

\section{Methodological quality assessment of RCTs}

Results of the methodological quality assessment of the seven included RCTs are shown in Table 3. Interrater agreement was excellent in the independent assessment process (kappa index of 0.82), and consensus was reached after discussion. Among the seven RCTs, six (85.7\%) were regarded to be of "high quality'. The major methodological quality issues with these RCTs were inappropriate concealed allocation (85.7\%), lack of blinded trial assessor (85.7\%), and lack of blinded therapists (71.4\%).

\section{Effectiveness of LLLT (PBM)}

Due to a limited number of eligible RCTs, only posttreatment and short-term follow-up comparisons ( $<6$ months after randomization) could be assessed. Subgroup analyses were conducted as planned: in total, comparisons of three control groups for primary and secondary outcomes were made as below. Table 4 summarizes the results of individual studies.

\section{LLLT (PBM) versus sham laser $(\boldsymbol{n}=3)$}

Three high quality studies [46-48] provided strong evidence that LLLT (PBM) was more effective than sham treatment for short-term (1 month post-treatment) total reduction in limb circumference. Two high quality studies $[46,48]$ provided conflicting evidence regarding the effects of LLLT (PBM) over sham laser on limb volume and shoulder mobility at the end of treatment. Two RCTs of high quality $[46,47]$ provided strong evidence suggesting similar effects from LLLT (PBM) and sham for range of movement in the affected limb in a shortterm follow-up. There was moderate evidence (based upon a single high quality study [47]) supporting the effectiveness of LLLT (PBM) over sham laser for pain relief in a short-term follow-up ( 2 months post treatment).

\section{LLLT (PBM) versus conventional therapy $(n=3)$}

Three high quality studies [49-51] provided conflicting evidence regarding differences between LLLT (PBM) and conventional therapy for short-term limb circumference reduction: two studies $[50,51]$ showed significant superior effects of LLLT (PBM) over compression (i.e. compression bandage and pneumatic compression) in limb girth at discharge; the other RCT [49] reported that
LLLT (PBM) did not significantly differ in results from manual lymphatic drainage at the end of treatment. There was moderate evidence (one high quality RCT [50]) that LLLT (PBM) and pneumatic compression therapy were not significantly different at a 3-month follow-up. For secondary outcome measures, only pain intensity was compared; however, findings from two studies (high quality) produced contradictory conclusions. LLLT (PBM) was significantly superior to compression bandage for pain relief post treatment [51], whereas no significant differences were detected at treatment termination when compared with pneumatic compression [50]. There was moderate evidence (one high quality RCT [50]) showing an equivalent reductions in pain intensity level from LLLT (PBM) and pneumatic compression therapy at a short-term follow-up (3 months post treatment).

\section{LLLT (PBM) versus a waiting list control $(n=1)$}

One RCT of low quality $(n=21)$ [52] found statistically significant effects of LLLT (PBM) in decreasing arm volume over no treatment at 4-weeks follow-up, yielding limited evidence in this comparison. However, no differences for such a comparison were found between these two groups immediately post-treatment (limited evidence).

\section{Application of LLLT (PBM)}

Treatment parameters of LLLT (PBM) extracted from all 11 studies included in the review, and are displayed in Table 5. The standard of reporting of the laser parameters in the included studies was poor and did not follow WALT recommendations [53]. The most common wavelength used was $904 \mathrm{~nm}$, reported in $6 / 11$ studies $[46,48-50,54,55]$, three studies used a combination of two wavelengths $[44,45,51]$, and one study failed to report the wavelength used [52]. When it was reported, the most common energy densities were $1.5 \mathrm{~J} / \mathrm{cm}^{2}[46-48,50]$ and $2.4 \mathrm{~J} / \mathrm{cm}^{2}$ $[44,45,51]$. The common sites of application were the cubital fossa and the axillary region. Regimes typically delivered 3 treatments per week with variation in the duration of treatment from 4 weeks to 12 weeks. Three studies provided shorter treatment cycles with an 8 week stand-down between cycles $[46,47,55]$.

\section{Discussion}

The primary aim of this systematic review was to evaluate the effectiveness of LLLT (PBM) in the management of BCRL. Findings support the use of LLLT (PBM) for treating women with BCRL. Based upon the best evidence synthesis, the current review provided strong evidence (three high quality trials) favoring LLLT (PBM) over sham in terms of reduction in limb edema at shortterm follow-up. For other comparisons, this review 


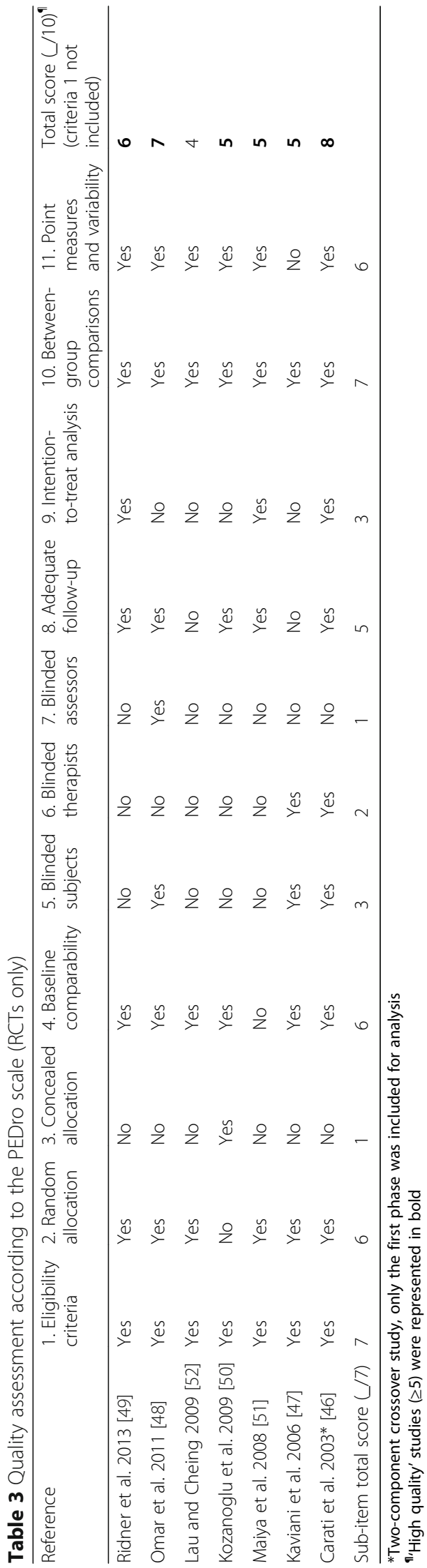


Table 4 Summary of results of RCTs included in subgroup analysis

\begin{tabular}{|c|c|c|c|c|c|c|}
\hline \multirow[t]{2}{*}{ Studies } & \multicolumn{2}{|c|}{$\underline{\text { Limb circumference/volume }}$} & \multicolumn{2}{|l|}{ Pain intensity } & \multicolumn{2}{|l|}{ Range of motion } \\
\hline & $\begin{array}{l}\text { Immediately after } \\
\text { end of all sessions }\end{array}$ & $\begin{array}{l}\text { Short-term } \\
\text { follow-up } \\
\text { (<6 months) }\end{array}$ & $\begin{array}{l}\text { Immediately after } \\
\text { end of all sessions }\end{array}$ & $\begin{array}{l}\text { Short-term } \\
\text { follow-up } \\
\text { (<6 months) }\end{array}$ & $\begin{array}{l}\text { Immediately after } \\
\text { end of all sessions }\end{array}$ & $\begin{array}{l}\text { Short-term } \\
\text { follow-up } \\
\text { (<6 months) }\end{array}$ \\
\hline \multicolumn{7}{|l|}{ LLLT (PBM) vs. sham laser } \\
\hline Omar et al. 2011 [48] & + & + & NR & NR & + & NR \\
\hline Kaviani et al. 2006 [47] & NR & $+^{*}$ & NR & + & NR & - \\
\hline Carati et al. 2003 [46] & - & $+^{*}$ & NR & NR & - & - \\
\hline \multicolumn{7}{|l|}{$\begin{array}{l}\text { LLLT (PBM) vs. conventional } \\
\text { therapy }\end{array}$} \\
\hline Ridner et al. 2013 [49] & - & NR & NR & NR & NR & NR \\
\hline Kozanoglu et al. 2009 [50] & + & - & - & - & NR & NR \\
\hline Maiya et al. 2008 [51] & + & $N R$ & + & NR & NR & NR \\
\hline \multicolumn{7}{|c|}{ LLLT (PBM) vs. a waiting list control } \\
\hline Lau and Cheing 2009 [52] & - & + & NR & NR & NR & NR \\
\hline
\end{tabular}

+: LLLT was more effective than the control group; -: LLLT was not more effective than the control group; ${ }^{*}$ comparison at 1 month post treatment LLLT low level laser therapy, PBM photobiomodulation, NR not reported

provided moderate evidence (one high quality trial) favoring LLLT (PBM) over sham for short-term pain relief, and limited evidence (one low quality trial) favoring LLLT (PBM) over no treatment for decreasing limb swelling at a short-term follow-up.

As a relatively novel therapeutic tool for the treatment of BCRL, LLLT (PBM) has gained increasing popularity since its approval by the United States Food and Drug Administration in 2007. Over the past two decades, seven RCTs [46-52] and four observational studies $[44,45,54,55]$ have been published in this area. Since RCTs are considered as the gold standard of contemporary medical research, the current systematic review generated conclusions about effectiveness of LLLT (PBM) based on the seven included RCTs. It is encouraging to note that the methodological quality of identified RCTs was 'high' in accordance with the PEDro scale (over 5/10); findings of this review were considered to be robust. Nevertheless, there was extensive study heterogeneity in treatment protocols, comparators, outcome measures, and follow-up periods. Due to a limited number of included studies, a head-to-head comparison to determine a superior LLLT (PBM) treatment regime was not possible. Future research into this area is suggested, which could provide evidence to guide development of an optimal LLLT (PBM) therapy regime for symptom management of BCRL.

This is the first systematic review applying best evidence synthesis to comprehensively evaluate the therapeutic value of LLLT (PBM) for BCRL. Findings from the review have strengthened conclusions of previous reviews $[15,36,37]$, and confirmed the effectiveness of LLLT (PBM) in the treatment of BCRL. While two previous reviews $[15,36]$ showed favorable results of LLLT (PBM) in reduction of limb volume and tissue hardness, it was argued that these reviews lacked formal analysis methodology, thus reliability of the conclusions was unclear. Smoot et al. conducted a meta-analysis [37] to synthesize evidence from intervention studies, and concluded that there was moderate-strength evidence supporting the use of LLLT (PBM) in the management of BCRL. Although this review was rated as 'moderate quality' (6/11) according to the Assessment of Multiple Systematic Reviews (AMSTAR) criteria (a validated instrument for quality assessment of systematic reviews) [56], clinical appropriateness of pooling study results irrespective of control comparisons (lack of subgroup analysis) may limit the validity of the review conclusions.

Sham laser was typically set as a control arm in the included RCTs. Although the use of sham laser well satisfied the methodology requirement of double blinding to investigate the specific effects of LLLT (PBM), rationale for clinical utility of a novel treatment intervention (for instance, LLLT (PBM)) is best demonstrated against an accepted standard (best) therapy. This review found conflicting evidence regarding the effectiveness of LLLT (PBM) over conventional treatments, including manual lymphatic drainage, pneumatic compression therapy and compression bandage [49-51], on limb circumference and pain intensity. Another systematic review evaluating a series of conservative therapies has demonstrated that LLLT (PBM) yielded a similar percentage of volume reductions (approximately 11\%) to compression garment or bandage [57]. Previous research suggested that wearing a compression garment alone results in a moderately significant reduction in BCRL [58]. Considering the intractable nature of $\mathrm{BCRL}$, an integrative treatment 





package, in which LLLT (PBM) is used in addition to compression garment, may be a reasonable clinical option, and deserves further investigation through welldesigned high quality RCTs.

Despite the clear statement by the WALT advocating standards of reporting of parameters when conducting studies involving laser therapy [53], there still seems to be inadequate information provided by authors of such studies. This is not uncommon and other systematic reviews have also highlighted these failures [59, 60]. Heterogeneity of the parameters used in the included studies and variable methods of application, along with differences in treatment regimes, all contribute to the difficulties of pooling information to make definitive statements regarding this use of LLLT (PBM) for BCRL. That being said, the normal genesis of treatment guidelines will result in many studies that show variation or contradictory results. Until patterns are recognized on a consistent basis across studies, the window of effective parameters cannot be identified. From all 11 studies included in this review, infrared wavelengths (808-905 $\mathrm{nm}$ ) have been most commonly employed to date, and reported energy densities in the range of $1.5 \mathrm{~J} / \mathrm{cm}^{2}-2.4 \mathrm{~J} / \mathrm{cm}^{2}$ have delivered positive outcomes. In comparison, effective energy densities for tendinopathy range from $1.8 \mathrm{~J} / \mathrm{cm}^{2}$ to $19.2 \mathrm{~J} / \mathrm{cm}^{2}$ depending upon the location of the tendon [59]. The reported frequency and duration of treatment is however too varied to make any strong statements, but a minimum of 4 weeks seems to be required.

The current review has adopted robust methodology to minimize the risk of bias. Firstly, it implemented most of the items listed in the AMSTAR checklist [56], therefore has a high methodological quality score (internal validity) of 9/11 (two points were missing because of the lack of a priori review protocol registry and an assessment of publication bias due to the qualitative analysis methodology). Secondly, in terms of the external validity, reporting of this review strictly adhered to the Preferred Reporting Items for Systematic Reviews and MetaAnalyses (PRISMA) guidelines [61] to ensure research replication. Thirdly, for data synthesis, subgroup analyses stratified by control comparisons and outcome measures were performed to address the influence of clinical (as well as statistical) heterogeneity. Fourthly, conclusions of the review were synthesized from seven RCTs with high methodology quality.

The primary limitation of this systematic review derived from the small number of included studies and lack of conclusions regarding the longer-term effects of LLLT (PBM) for BCRL management. Findings of this review suggest future well-designed fully powered RCTs are needed to inform the superiority of different LLLT (PBM) interventions, and determine an optimal treatment protocol for this therapy.

\section{Conclusions}

Based upon the current systematic review, LLLT (PBM) in the management of BCRL is more effective for limb edema reduction than sham and no treatment at a short-term follow-up, and not more effective than other conventional treatments. Data suggest that LLLT (PBM) may be an effective treatment approach for women with BCRL. Due to the limited numbers of published trials available, there is a clear need for well-designed highquality trials in this area. The optimal treatment parameters for clinical application have yet to be elucidated.

\section{Additional files}

Additional file 1: Search strategy (PDF). This file presents the search strategy used in this systematic review in four databases (PubMed, AMED, Web of Science and CNKI). (PDF $193 \mathrm{~kb}$ )

Additional file 2: Excluded articles after duplicates removal $(n=66)$ (PDF). This file presents references of the 66 articles that were excluded after duplicates removed. (DOCX $23 \mathrm{~kb}$ )

\section{Abbreviations}

AMSTAR: Assessment of Multiple Systematic Reviews; ATP: Adenosine triphosphate; BCRL: Breast cancer related lymphedema; LLLT: Low level laser therapy; NO: Nitric oxide; PBM: Photobiomodulation; PRISMA: Preferred Reporting Items for Systematic Reviews and Meta-Analyses; RCT: Randomized controlled trials; REDOX: Reduction-oxidation reaction; ROS: Reactive oxygen species; WALT: World Association for Laser Therapy

\section{Acknowledgements}

Not applicable.

\section{Funding}

This work was funded by the New Zealand Breast Cancer Foundation. The Foundation had no role in this manuscript other than providing funding support.

\section{Availability of data and materials}

The datasets supporting the conclusions of this article are included within the article and its additional files.

\section{Authors' contributions}

GDB: principal investigator; review concept and design; drafting and revision; review of treatment parameters; LL: review design, literature search and selection, data extraction and methodological quality assessment, data analysis and interpretation, drafting and revision; ASG: literature search and selection, data extraction and methodological quality assessment; CC, SP, and JJA: revision; ST: drafting and revision; review of treatment parameters. All authors read and approved the final manuscript.

Ethics approval and consent to participate

Not applicable.

Consent for publication

Not applicable.

Competing interests

The authors declare that they have no competing interests.

\section{Publisher's Note}

Springer Nature remains neutral with regard to jurisdictional claims in published maps and institutional affiliations. 


\section{Author details}

'Centre for Health, Activity and Rehabilitation Research, School of Physiotherapy, University of Otago, Dunedin, New Zealand. ${ }^{2}$ Department of Surgical Sciences, Southern District Health Board, Dunedin, New Zealand. ${ }^{3}$ Department of Anatomy, Physiology and Genetics, Uniformed Services University of the Health Sciences, Maryland, MD, USA.

\section{Received: 21 May 2017 Accepted: 28 November 2017}

\section{Published online: 07 December 2017}

\section{References}

1. Pinto AC, de Azambuja E. Improving quality of life after breast cancer: dealing with symptoms. Maturitas. 2011;70(4):343-8.

2. The New Zealand Breast Cancer Foundation Breast Cancer Overview. http:// nzbcf.org.nz/BREASTCANCER/Overview/BreastCancerinNZ/Fastfacts.aspx. Accessed 01 Nov 2016

3. DiSipio T, Rye S, Newman B, Hayes S. Incidence of unilateral arm lymphoedema after breast cancer: a systematic review and meta-analysis. Lancet Oncol. 2013;14(6):500-15.

4. Asim M, Cham A, Banerjee S, Nancekivell R, Dutu G, McBride C, Cavanagh S, Lawrenson R, Campbell I. Difficulties with defining lymphoedema after axillary dissection for breast cancer. NZ Med J. 2012;125(1351):29-39.

5. Morrell RM, Halyard MY, Schild SE, Ali MS, Gunderson LL, Pockaj BA. Breast cancer-related lymphedema. Mayo Clin Proc. 2005:80(11):1480-4.

6. Ridner SH, Dietrich MS, Kidd N. Breast cancer treatment-related lymphedema self-care: education, practices, symptoms, and quality of life. Support Care Cancer. 2011;19(5):631-7.

7. Park JE, Jang HJ, Seo KS. Quality of life, upper extremity function and the effect of lymphedema treatment in breast cancer related lymphedema patients. Ann Rehabil Med. 2012;36(2):240-7.

8. Penha TR, Botter B, Heuts EM, Voogd AC, von Meyenfeldt MF, van der Hulst RR. Quality of life in patients with breast cancer-related lymphedema and reconstructive breast surgery. J Reconstr Microsurg. 2016;32(6):484-90.

9. International Society of Lymphology. The diagnosis and treatment of peripheral lymphedema: 2013 consensus document of the International Society of Lymphology. Lymphology. 2013;46(1):1-11

10. Oremus M, Dayes I, Walker K, Raina P. Systematic review: conservative treatments for secondary lymphedema. BMC Cancer. 2012;12:6.

11. Nouri K, Jimenez GP, Harrison-Balestra C, Elgart GW. 585-nm pulsed dye laser in the treatment of surgical scars starting on the suture removal day. Dermatol Surg. 2003;29(1):65-73. discussion 73

12. Lievens PC. The effect of a combined HeNe and i.R. Laser treatment on the regeneration of the lymphatic system during the process of wound healing. Lasers Med Sci. 1991;6(2):193-9.

13. Assis L, Moretti Al, Abrahao TB, de Souza HP, Hamblin MR, Parizotto NA. Low-level laser therapy $(808 \mathrm{~nm}$ ) contributes to muscle regeneration and prevents fibrosis in rat tibialis anterior muscle after cryolesion. Lasers Med Sci. 2013:28(3):947-55

14. Jang DH, Song DH, Chang EJ, Jeon JY. Anti-inflammatory and lymphangiogenetic effects of low-level laser therapy on lymphedema in an experimental mouse tail model. Lasers Med Sci. 2016;31(2):289-96.

15. Lima MT E, Lima JG E, de Andrade MF, Bergmann A. Low-level laser therapy in secondary lymphedema after breast cancer: Systematic review. Lasers Med Sci. 2014:29(3):1289-95.

16. Chen $\mathrm{CH}$, Tsai JL, Wang YH, Lee $\mathrm{CL}$, Chen JK, Huang MH. Low-level laser irradiation promotes cell proliferation and mRNA expression of type I collagen and decorin in porcine Achilles tendon fibroblasts in vitro. J Orthop Res. 2009;27(5):646-50.

17. Gavish L, Perez L, Gertz SD. Low-level laser irradiation modulates matrix metalloproteinase activity and gene expression in porcine aortic smooth muscle cells. Lasers Surg Med. 2006;38(8):779-86.

18. Huang Y-Y, Chen ACH, Sharma SK, Wu Q, Hamblin MR. Comparison of cellular responses induced by low level light in different cell types. Proc SPIE. 2010;7552

19. Kreisler M, Christoffers AB, Willershausen B, d'Hoedt B. Effect of low-leve GaAlAs laser irradiation on the proliferation rate of human periodontal ligament fibroblasts: an in vitro study. J Clin Periodontol. 2003;30(4):353-8.

20. Peplow PV, Chung TY, Baxter GD. Laser photobiomodulation of wound healing: a review of experimental studies in mouse and rat animal models. Photomed Laser Surg. 2010;28(3):291-325
21. Stadler I, Evans R, Kolb B, Naim JO, Narayan V, Buehner N, Lanzafame RJ. In Vitro effects of low-level laser irradiation at $660 \mathrm{~nm}$ on peripheral blood lymphocytes. Lasers Surg Med. 2000;27(3):255-61.

22. Stein A, Benayahu D, Maltz L, Oron U. Low-level laser irradiation promotes proliferation and differentiation of human osteoblasts in vitro. Photomed Laser Surg. 2005:23(2):161-6.

23. Tuby H, Maltz L, Oron U. Low-level laser irradiation (LLLI) promotes proliferation of mesenchymal and cardiac stem cells in culture. Lasers Surg Med. 2007;39(4):373-8

24. Vinck EM, Cagnie BJ, Cornelissen MJ, Declercq HA, Cambier DC. Increased fibroblast proliferation induced by light emitting diode and low power laser irradiation. Lasers Med Sci. 2003;18(2):95-9.

25. Karu T: Ten lectures on basic science of laser phototherapy. Sweden: Graengesberg: Prima Books; 2007

26. Gavish L, Perez LS, Reissman P, Gertz SD. Irradiation with $780 \mathrm{~nm}$ diode laser attenuates inflammatory cytokines but upregulates nitric oxide in lipopolysaccharide-stimulated macrophages: implications for the prevention of aneurysm progression. Lasers Surg Med. 2008;40(5):371-8.

27. Hou JF, Zhang H, Yuan X, Li J, Wei YJ, SS H. In Vitro effects of low-level laser irradiation for bone marrow mesenchymal stem cells: proliferation, growth factors secretion and myogenic differentiation. Lasers Surg Med. 2008; 40(10):726-33.

28. Rocha Junior AM, Vieira BJ, de Andrade LC, Aarestrup FM. Low-level laser therapy increases transforming growth factor-beta2 expression and induces apoptosis of epithelial cells during the tissue repair process. Photomed Laser Surg. 2009;27(2):303-7.

29. Saygun I, Karacay S, Serdar M, Ural AU, Sencimen M, Kurtis B. Effects of laser irradiation on the release of basic fibroblast growth factor (bFGF), insulin like growth factor-1 (IGF-1), and receptor of IGF-1 (IGFBP3) from gingival fibroblasts. Lasers Med Sci. 2008:23(2):211-5.

30. Gao X, Xing D. Molecular mechanisms of cell proliferation induced by low power laser irradiation. J Biomed Sci. 2009;16:4.

31. Hawkins DH, Abrahamse $\mathrm{H}$. The role of laser fluence in cell viability, proliferation, and membrane integrity of wounded human skin fibroblasts following helium-neon laser irradiation. Lasers Surg Med. 2006;38(1):74-83.

32. Karu T. Primary and secondary mechanisms of action of visible to near-IR radiation on cells. J Photochem Photobiol B. 1999:49(1):1-17.

33. Lievens $P$ : The Influence of laser irradiation on the motricity of lymphatical system and on the wound healing process. In: International Congress on Laser in Medicine and Surgery: 26-28 June 1985; Bologna; 1985: 171-174.

34. Inoue K, Nishioka J, Hukuda S. Altered lymphocyte proliferation by low dosage laser irradiation. Clin Exp Rheumatol. 1989;7(5):521-3.

35. Horvath Z, et al. Possible ab-initio explanation of laser "biostimulation" effects Laser applications in medicine and surgery. Edited G. Galetti et al. Proc 3rd World Congr - Intl Soc Low Power Laser Appln in Medicine. 1992. p. 57.

36. Omar MT, Shaheen AA, Zafar H. A systematic review of the effect of lowlevel laser therapy in the management of breast cancer-related lymphedema. Support Care Cancer. 2012:20(11):2977-84.

37. Smoot B, Chiavola-Larson L, Lee J, Manibusan H, Allen DD. Effect of low level laser therapy on pain and swelling in women with breast cancerrelated lymphedema: a systematic review and meta-analysis. J Cancer Surviv. 2015:9(2):287-304

38. Verhagen AP, de Vet HC, de Bie RA, Kessels AG, Boers M, Bouter LM, Knipschild PG. The Delphi list: a criteria list for quality assessment of randomized clinical trials for conducting systematic reviews developed by Delphi consensus. J Clin Epidemiol. 1998;51(12):1235-41.

39. Fleiss JL. Measuring nominal scale agreement among many raters. Psychol Bull. 1971;76(5):378-82

40. Moseley AM, Herbert RD, Sherrington C, Maher CG. Evidence for physiotherapy practice: a survey of the physiotherapy evidence database (PEDro). Aust J Physiother. 2002:48(1):43-9.

41. Karki A, Anttila H, Tasmuth T, Rautakorpi UM. Lymphoedema therapy in breast cancer patients: a systematic review on effectiveness and a survey of current practices and costs in Finland. Acta Oncol. 2009;48(6):850-9.

42. van Tulder M, Furlan A, Bombardier C, Bouter L. Updated method guidelines for systematic reviews in the cochrane collaboration back review group. Spine (Phila Pa 1976). 2003:28(12):1290-9.

43. Recommended treatment doses for low level laser therapy. http://waltza.co. $\mathrm{za} /$ documentation-links/recommendations/dosage-recommendations/. Accessed 01 Nov 2016 
44. Piller NB, Thelander A. Treating chronic post-mastectomy lymphoedema with low level laser therapy: a cost effective strategy to reduce severity and improve the quality of survival. Laser Ther. 1995;7(4):163-8.

45. Piller NB, Thelander A. Treatment of chronic postmastectomy lymphedema with low level laser therapy: a 2.5 year follow-up. Lymphology. 1998;31(2):74-86.

46. Carati CJ, Anderson SN, Gannon BJ, Piller NB. Treatment of postmastectomy lymphedema with low-level laser therapy: a double blind, placebocontrolled trial. Cancer. 2003;98(6):1114-22.

47. Kaviani A, Fateh M, Yousefi-Nooraie R, Alinagi-Zadeh MR, Ataie-Fashtami L. Low-level laser therapy in management of postmastectomy lymphedema. Lasers Med Sci. 2006;21(2):90-4.

48. Omar MTA, Ebid AA, El Morsy AM. Treatment of post-mastectomy lymphedema with laser therapy: double blind placebo control randomized study. J Surg Res. 2011;165(1):82-90

49. Ridner SH, Poage-Hooper E, Kanar C, Doersam JK, Bond SM, Dietrich MS. A pilot randomized trial evaluating low-level laser therapy as an alternative treatment to manual lymphatic drainage for breast cancer-related lymphedema. Oncol Nurs Forum. 2013;40(4):383-93.

50. Kozanoglu E, Basaran S, Paydas S, Sarpel T. Efficacy of pneumatic compression and low-level laser therapy in the treatment of postmastectomy lymphoedema: a randomized controlled trial. Clin Rehabil. 2009;23(2):117-24.

51. Maiya A, Olivia E, Dibya A. Effect of low energy laser therapy in the management of post- mastectomy lymphoedema. Physiotherapy Singapore. 2008;11(1):2-5.

52. Lau RW, Cheing GL. Managing postmastectomy lymphedema with low-level laser therapy. Photomed Laser Surg. 2009;27(5):763-9.

53. World Association of Laser Therapy (WALT). Consensus agreement on the design and conduct of clinical studies with low-level laser therapy and light therapy for musculoskeletal pain and disorders. Photomed Laser Surg. 2006; 24(6):761-2.

54. Mayrovitz HN, Davey S. Changes in tissue water and indentation resistance of lymphedematous limbs accompanying low level laser therapy (LLLT) of fibrotic skin. Lymphology. 2011;44(4):168-77.

55. Dirican A, Andacoglu O, Johnson R, McGuire K, Mager L, Soran A. The shortterm effects of low-level laser therapy in the management of breast-cancerrelated lymphedema. Support Care Cancer. 2011;19(5):685-90.

56. Shea BJ, Grimshaw JM, Wells GA, Boers M, Andersson N, Hamel C, Porter AC, Tugwell P, Moher D, Bouter LM. Development of AMSTAR: a measurement tool to assess the methodological quality of systematic reviews. BMC Med Res Methodol. 2007;7:10

57. Moseley AL, Carati CJ, Piller NB. A systematic review of common conservative therapies for arm lymphoedema secondary to breast cancer treatment. Ann Oncol. 2007;18(4):639-46.

58. Stout NL, Pfalzer LA, Springer B, Levy E, McGarvey CL, Danoff JV, Gerber LH, Soballe PW. Breast cancer-related lymphedema: comparing direct costs of a prospective surveillance model and a traditional model of care. Phys Ther. 2012;92(1):152-63.

59. Tumilty S, Munn J, McDonough S, Hurley DA, Basford JR, Baxter GD. Low level laser treatment of tendinopathy: a systematic review with metaanalysis. Photomed Laser Surg. 2010;28(1):3-16.

60. Law D, McDonough S, Bleakley C, Baxter GD, Tumilty S. Laser acupuncture for treating musculoskeletal pain: a systematic review with meta-analysis. $J$ Acupunct Meridian Stud. 2015;8(1):2-16.

61. Moher D, Liberati A, Tetzlaff J, Altman DG. Preferred reporting items for systematic reviews and meta-analyses: the PRISMA statement. Ann Intern Med. 2009;151(4):264-9. w264

\section{Submit your next manuscript to BioMed Central and we will help you at every step:}

- We accept pre-submission inquiries

- Our selector tool helps you to find the most relevant journal

- We provide round the clock customer support

- Convenient online submission

- Thorough peer review

- Inclusion in PubMed and all major indexing services

- Maximum visibility for your research

Submit your manuscript at www.biomedcentral.com/submit
Biomed Central 